\title{
Chimera and phase-cluster states in populations of coupled chemical oscillators
}

\author{
Mark R. Tinsley, Simbarashe Nkomo and Kenneth Showalter ${ }^{\star}$
}

\begin{abstract}
Populations of coupled oscillators may exhibit two coexisting subpopulations, one with synchronized oscillations and the other with unsynchronized oscillations, even though all of the oscillators are coupled to each other in an equivalent manner. This phenomenon, discovered about ten years ago in theoretical studies ${ }^{1}$, was then further characterized ${ }^{2}$ and named the chimera state after the Greek mythological creature made up of different animals. The highly counterintuitive coexistence of coherent and incoherent oscillations in populations of identical oscillators, each with an equivalent coupling structure, inspired great interest and a flurry of theoretical activity $^{3-19}$. Here we report on experimental studies of chimera states and their relation to other synchronization states in populations of coupled chemical oscillators. Our experiments with coupled Belousov-Zhabotinsky oscillators ${ }^{20,21}$ and corresponding simulations reveal chimera behaviour that differs significantly from the behaviour found in theoretical studies of phase-oscillator models.
\end{abstract}

Following ref. 5, we study a system with two subpopulations of oscillators that are globally coupled, both within and between the subpopulations. As in the original model of ref. 1, in which each oscillator has an equivalent coupling structure, with coupling strength decreasing exponentially with distance, each oscillator in the model in ref. 5 has an equivalent coupling structure, with stronger global coupling to its fellow subpopulation members and weaker global coupling to the oscillators in the other subpopulation. Both model systems are multistable, with the fully synchronized state exhibited for homogeneous initial conditions and the chimera state exhibited for particular heterogeneous initial conditions $s^{4,5}$.

There are two sources of heterogeneity in coupled oscillator systems that exhibit chimera states. The nonlocal coupling of each oscillator is by its nature heterogeneous, such as two different coupling strengths in the model of ref. 5 or an exponentially decreasing coupling strength in the model of ref. 1. In addition, the coupling in a chimera state at any moment is heterogeneous in the sense that the coupling strength depends on the phase of each oscillator with respect to the phases of all the oscillators to which it is coupled ${ }^{1}$.

Our experiments are carried out with discrete chemical oscillators based on the photosensitive Belousov-Zhabotinsky reaction $^{20-22}$, in which the $\mathrm{Ru}(\mathrm{bpy})_{3}{ }^{2+}$ catalyst is loaded onto ion-exchange particles that are then immersed in a catalyst-free reaction mixture. The $N$ oscillatory particles are divided into two groups of equal size, $\mathrm{A}$ and $\mathrm{B}$, with each member $i$ of group $\sigma$ experiencing the feedback light intensity $P_{i}^{\sigma}$ :

$$
\begin{aligned}
P_{i}^{\sigma}= & P_{0}+k_{\sigma} P_{\max }\left(\hat{I}_{\sigma}(t-\tau)-I_{i}(t)\right) \\
& +k_{\sigma \sigma^{\prime}} P_{\max }\left(\hat{I}_{\sigma^{\prime}}(t-\tau)-I_{i}(t)\right)
\end{aligned}
$$

where $\sigma=\mathrm{A}, \mathrm{B}$ and $\sigma^{\prime}=\mathrm{B}, \mathrm{A}$. The mean intensity of the oscillators in group $\sigma$ is $\hat{I}_{\sigma}$, and $k_{\sigma}$ and $k_{\sigma \sigma^{\prime}}$ are the intra- and inter-group coupling coefficients, respectively, with $\left|k_{\sigma \sigma^{\prime}}\right|<k_{\sigma}$. Each member of each group is globally coupled through the mean signal of its own group and the mean signal of the other group, with the intra-group coupling stronger than the inter-group coupling. The maximum light perturbation is $P_{\max }=3.0 \mathrm{~mW} \mathrm{~cm}^{-2}$, the background light intensity is $P_{0}=1.4 \mathrm{~mW} \mathrm{~cm}^{-2}$ and the measured intensity $I$ is scaled from 0 to 1 . The delay term $\tau$ (refs $6,11,23$ ) plays a role akin to the phase-frustration term used in the model in ref. 5 and in other phase-oscillator models of chimera states.

This coupling scheme gives rise to several different types of synchronization behaviour in our experiments. In all cases, group A remains fully synchronized, whereas group B exhibits four basic states: fully synchronized, $n$-cluster, the unsynchronized chimera and semi-synchronized. We label these states $1-1,1-n$ $(n=2,3,4, \ldots), 1-\mathrm{c}$ and $1-\mathrm{s}$, respectively, where the first number represents the state of group A and the second number (or letter) represents the state of group $B$.

During full synchronization, the 1-1 state, members of group B synchronize both in frequency and phase $\mathrm{e}^{23}$, typically with a constant phase difference relative to the synchronized members of group A. Figure 1a shows a time series of the mean oscillator intensity in groups $\mathrm{A}$ and $\mathrm{B}$, and the inset shows a snapshot of the phase of each oscillator in the two groups. An example of a two-cluster state ${ }^{24}$ is shown in Fig. 1b, where the different occupancies of the two clusters are visible in the phase snapshot. We see a wide range of occupancies in the two-cluster state as well as in higher order 1-3 and 1-4 states in our experiments.

In the 1-c chimera state, the members of group B typically remain unsynchronized for the entire duration of the experiment. The mean intensity for group B is a small-amplitude, noisy signal, as shown in Fig. 1c. At any moment, the phases of the oscillators are spread almost evenly between 0 and $2 \pi$ (see inset). In addition to chimera states, we also observe semi-synchronized states, which involve higher- and lower-frequency oscillators intermittently aligning, resulting in occasional mean intensities with irregular large amplitudes (Fig. 1d). An example of transient partial alignment leading to larger values in mean amplitude is shown in the phase snapshot. The behaviours shown in Fig. 1 are found at different intra- and inter-group coupling strengths, and the various behaviour regimes as a function of $k_{\mathrm{A}}=k_{\mathrm{B}}$ and $k_{\mathrm{AB}}=k_{\mathrm{BA}}$ are shown in Fig. 2a. The synchronized 1-1 state is in-phase or out-of-phase for $k_{\mathrm{AB}}>0$ or $k_{\mathrm{AB}}<0$, respectively.

The 1-c chimera region shown in Fig. 2a occurs primarily with the inter-group coupling strength $k_{\mathrm{AB}}<0$, although there is a small region of $1-\mathrm{c}$ behaviour for $k_{\mathrm{AB}}>0$. The chimera for negative inter-group coupling strengths was surprising in view of the model of ref. 5, which employs only positive coupling, and we therefore investigated the underlying coupling mechanism. Figure $2 \mathrm{~b}$ shows experimental and simulated phase-response curves for perturbations involving both increases and decreases in light 

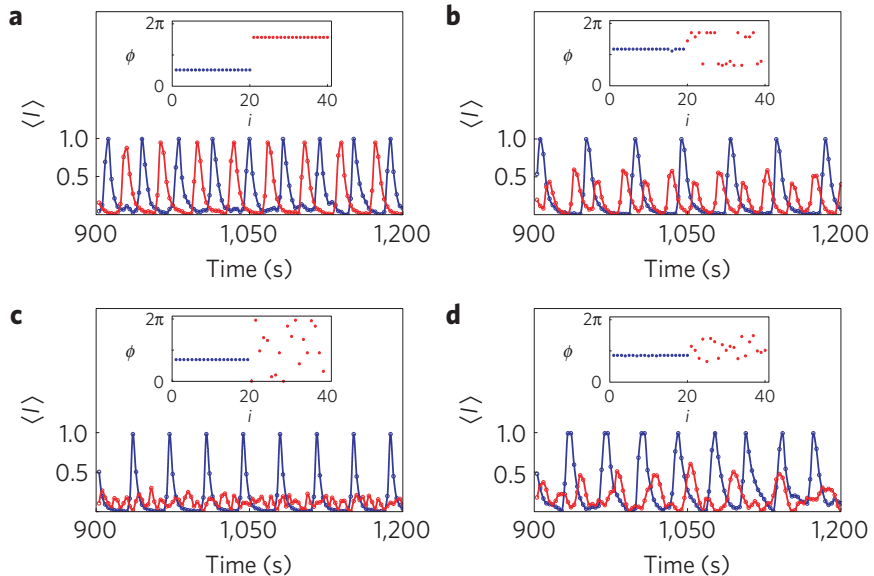

Figure $1 \mid$ Normalized mean intensity $\langle I\rangle$ of group A and group B as a function of time, and snapshot of the phases $\phi$ of oscillators $i=1-20$ in group $A$ and 21-40 in group B. Blue, group $A$; red, group B. a, Synchronized out-of-phase 1-1 state, $k_{A}=3.0, k_{A B}=-1.8$. b, Synchronized 1-2 phase cluster state, $k_{A}=4.0, k_{A B}=-1.0$. c Chimera 1-c state, $k_{A}=3.0$, $k_{A B}=-0.2$. d, Semi-synchronized 1-s state, $k_{A}=2.0, k_{A B}=-1.0$. All time-series curves show fits to data points (o). The phase $\phi=0-2 \pi$ of an individual oscillator $i$ (inset) is assigned by linear interpolation between adjacent maxima. In all of the experiments $\tau=30 \mathrm{~s}$.

intensity. As both positive and negative perturbations give rise to positive phase responses, both positive and negative values of $k_{\mathrm{AB}}$ result in positive inter-group coupling, consistent with the theoretical model ${ }^{5}$.

We model the experimental system with a two-variable mode ${ }^{25}$ for the photosensitive Belousov-Zhabotinsky particle system ${ }^{21}$, where $\mathrm{d} X_{i} / \mathrm{d} t=f\left(X_{i}, Z_{i}, q_{i}\right)+\phi_{i}^{\sigma}, \mathrm{d} Z_{i} / \mathrm{d} t=g\left(X_{i}, Z_{i}, q_{i}\right)+2 \phi_{i}^{\sigma}$ describes the chemistry of each oscillator $i$ in group $\sigma$ (see Supplementary Information). The terms $f$ and $g$ give the nonphotochemical components of the Belousov-Zhabotinsky reaction, and $X_{i}, Z_{i}$ and $q_{i}$ are $\left[\mathrm{HBrO}_{2}\right],\left[\mathrm{Ru}(\mathrm{bpy})_{3}{ }^{3+}\right]$ and the stoichiometric coefficient, respectively, associated with the $i$ th oscillator.

The photo-excitatory feedback to oscillator $i$ in group $\sigma$ is $\phi_{i}^{\sigma}$, calculated according to

$$
\begin{aligned}
\phi_{i}^{\sigma}= & \phi_{0}+k_{\sigma} \phi_{\max }\left(\hat{Z}_{\sigma}(t-\tau)-Z_{i}(t)\right) \\
& +k_{\sigma \sigma^{\prime}} \phi_{\max }\left(\hat{Z}_{\sigma^{\prime}}(t-\tau)-Z_{i}(t)\right)
\end{aligned}
$$

with other terms as in equation (1). Simulations are carried out with up to 80 particles (40 per group) for both homogeneous (period $=41.0)$ and heterogeneous (period $=41.0 \pm 2.1$ ) oscillator systems. The heterogeneous oscillator system has an approximately normal distribution in period.

The model simulations generate behaviour in qualitative agreement with that exhibited by the experimental system, namely, the 1-1, 1- $n, 1-c$ and 1-s states. Standard continuation methods were used to determine whether or not a state is stable in a given parameter region and whether other stable states coexist. The location of these states in the $k_{\mathrm{A}}-k_{\mathrm{AB}}$ phase diagram (see Supplementary Information) is similar to the experimental behaviour shown in Fig. 2a, with a small region of chimera behaviour observed for $k_{\mathrm{AB}}>0$ and a much larger region observed for $k_{\mathrm{AB}}<0$.

The probability of the homogeneous frequency system residing in the 1-c, 1-1 or 1-2 state as a function of $k_{\mathrm{A}}$ is shown in Fig. 2c. At low values of $k_{\mathrm{A}}$, the system predominantly finds the chimera state, with the 1-1 and 1-2 states increasingly prevalent at higher values of $k_{\mathrm{A}}$. Continuity methods demonstrate that the chimera is embedded
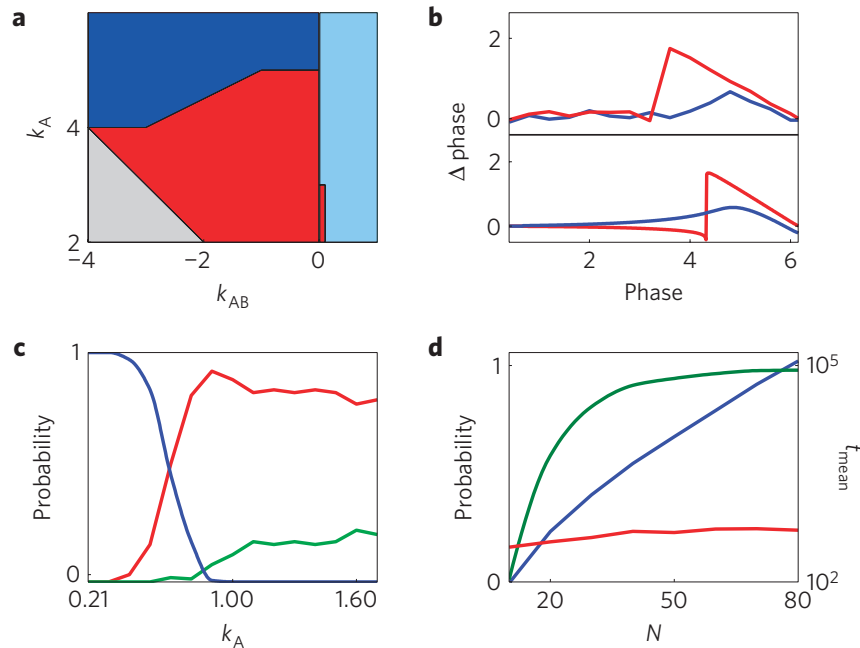

Figure 2 | Dependence of the chimera and cluster states on parameters. a, Regions of 1-c chimera behaviour (red), 1-1 in-phase (light blue) and 1-1 out-of-phase (dark blue) synchronization observed in experiments as a function of coupling strengths $k_{A}$ and $k_{A B}$. The grey region corresponds to values of $\left|k_{A B}\right|>k_{A} \cdot \mathbf{b}$, Phase-response curves for experimental (upper panel) and model (lower panel) systems, in which perturbations were an increase (red) or decrease (blue) in light intensity. c, Probability of 1-c (blue), 1-1 (red) and 1-2 (green) states as a function of $k_{\mathrm{A}}$ from simulations of the homogeneous frequency model, with $N=80$. Continuity methods yield stable states in the following regions: 1-1 state stable for all values of $k_{\mathrm{A}}, 1-2$ state stable for $0.23<k_{\mathrm{A}}<1.6,1-\mathrm{c}$ state stable for $0.21<k_{\mathrm{A}}<0.8$. d, Probability of a stable chimera state (green) for the homogeneous frequency system as a function of the number of oscillators $N$. The logarithm of the mean time to collapse to a synchronized state for homogeneous (red) and heterogeneous (blue) frequency oscillator populations is shown. Probabilities in $\mathbf{c}$ and $\mathbf{d}$ are based on 400 simulations for each abscissa value using random initial phases for group $B$; conditions: $\tau=36, k_{A}=0.6, k_{A B}=0.12$, except where otherwise indicated.

in a region of multistability, with the 1-1 state occurring over the entire range of $k_{\mathrm{A}}$ and small regions of higher-order 1-2, 1-3 and 1-4 cluster states also occurring. Non-stationary (breathing) chimera states were also found in certain parameter regimes, similar to those reported in ref. 5. No semi-synchronized behaviour is found in the homogeneous frequency system, because when partial phase alignment occurs, the system quickly relaxes to a 1-1 or 1- $n$ state. In the simulations for Fig. 2c, for example, the system rapidly evolved to a 1-1 or 1-2 state when the mean signal of group B exceeded a threshold (see Methods), indicating partial alignment.

Simulations were also carried out to determine the effects of group size and frequency heterogeneity on the probability of the system residing in the 1-c chimera state. For the homogeneous frequency system, the probability of the 1-c state increases with increasing group size, as shown in Fig. 2 d, where the 1-1 state is exhibited when the chimera state is not found. Also shown in Fig. $2 \mathrm{~d}$ is the lifetime of asynchronous states that collapsed to synchronized states. The relatively short lifetime of these collapsing states is almost constant, and they apparently arise from initial conditions unfavorable to finding the chimera state. In contrast, the system with a distribution of oscillatory frequencies exhibits chimera lifetimes that increase approximately exponentially with increasing system size, Fig. 2 d. No asymptotically stable chimera is observed in this system for this level of period heterogeneity $(41.0 \pm 2.1)$; however, extremely long-lived 1-c states are observed for larger system sizes $\left(>10^{4}\right.$ periods for $\left.N=70\right)$.

The small-amplitude mean signal of the chimera in the homogeneous frequency system is illustrated in Fig. 3a, as well as 

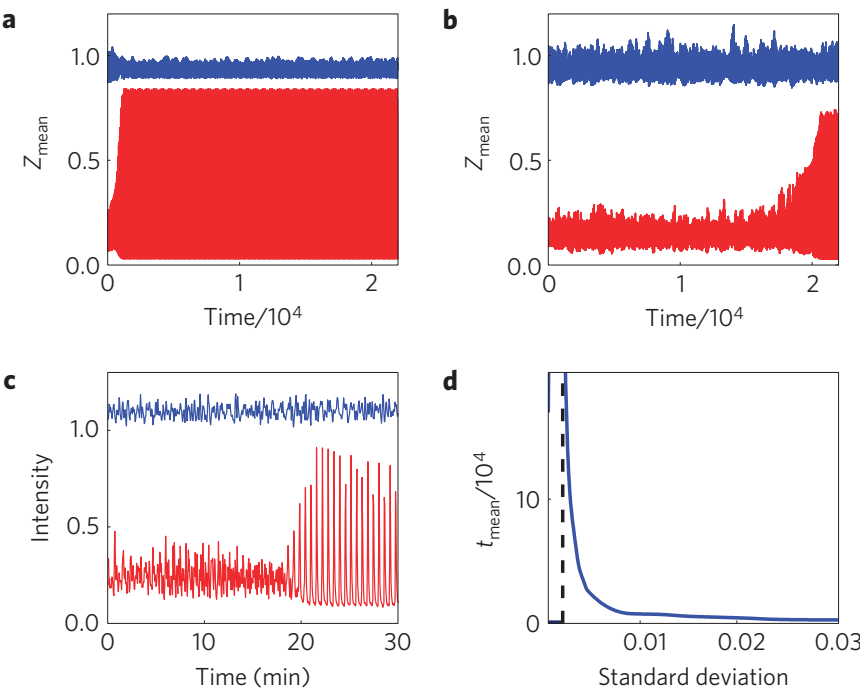

Figure 3 | Long-lived and transient chimera states in simulations and experiments. a, Time series for a chimera state (blue) and transient to a 1-1 state (red) in the homogeneous frequency model, with $N=60$. b. A chimera state (blue) and a collapse to synchronization (red) in the heterogeneous frequency model, with $N=60$. c A sustained chimera state (blue) and an example of a collapse to synchronization (red) in an experiment, with $k_{A}=3.0$ and $k_{A B}=-1.0$ in both cases. $\mathbf{d}$, Time to collapse in the heterogeneous frequency model as a function of the standard deviation of the parameter $q$ determining the period, with $N=40$. The dashed line shows the approximate location of the asymptote of the divergent chimera lifetime. Other parameters for $\mathbf{a}, \mathbf{b}$ and $\mathbf{d}$ are the same as in Fig. 2. The time series shown in $\mathbf{a}-\mathbf{c}$ are the mean signal for group B, with the blue time series offset for illustration purposes.

the large-amplitude mean signal of the system when the initial conditions lead quickly to a synchronized 1-1 state. Figure $3 \mathrm{~b}$ shows a long-lived chimera in the heterogeneous frequency system, with a noisy small-amplitude mean signal, along with the collapse of a chimera to a synchronized 1-1 state for the same conditions but with a different initial phase distribution. Figure $3 \mathrm{c}$ shows a long-lived chimera state along with a chimera collapsing to a synchronized 1:1 state in experiments with the same conditions.

The effects of period heterogeneity on the chimera lifetime can be seen in a plot of mean lifetime as a function of the standard deviation of the period distribution, shown in Fig. 3d. The mean lifetime markedly increases at low heterogeneity of the oscillator population. The simulations indicate that there is a small but finite range of very low period heterogeneity in which the chimera states are asymptotically stable.

Our studies of coupled chemical oscillators show that the heterogeneous frequency system, corresponding to the experiments and associated simulations, exhibits chimera, cluster and fully synchronized states. The chimera lifetime grows approximately exponentially with system size (Fig. 2d), and we find evidence of asymptotically stable chimera behaviour at very low levels of frequency heterogeneity (Fig. 3d). Interestingly, simulations of the homogeneous frequency system indicate that asymptotically stable chimera states occur and increase in occurrence with increasing system size (Fig. 2d.) This is in contrast with recent studies ${ }^{17}$, which demonstrate that the chimera state is transient for finite system sizes for identical phase oscillators. This suggests that the relaxation oscillations of the Belousov-Zhabotinsky system lend stability to the chimera state, as this is the primary feature that differs between these systems. We note that extremely long-lived chimera states in our simulations do not provide unequivocal evidence for asymptotic stability of these states.
The chimera behaviour found in our experiments and corresponding simulations of the heterogeneous frequency system is similar in many respects to the behaviour found in ref. 17 in a homogeneous phase-oscillator model. In this paper, it is pointed out that above small system sizes, the chimera state becomes very long-lived, and the behaviour is much like that observed in the thermodynamic limit, $N \rightarrow \infty$.

The chimera state in populations of coupled chemical oscillators is made up of subpopulations that exhibit coherent and incoherent oscillations. The chimera state arises from the non-local coupling and would disappear with uniform global coupling. The chimera behaviour is not the result of frequency heterogeneity in our system, because, in fact, frequency heterogeneity reduces the lifetime and hence the likelihood of finding the state (Fig. 3d). Chemical oscillators are stiff, relaxation oscillators, which may give rise to important differences in their chimera behaviour from the more idealized theoretical models. The semi-synchronized state in this system remains to be fully characterized, but we believe it may be the result of transient phase clusters (switchers) that are known to occur in related globally coupled Belousov-Zhabotinsky systems ${ }^{21,26}$.

The very existence of the chimera is a surprise, and we note that this peculiar dynamical behaviour could be of importance in certain biological and physical systems. Many birds as well as dolphins sleep with one eye open, in the sense that one hemisphere of the brain is synchronous while the other is asynchronous ${ }^{5,27}$. In addition, the simultaneous appearance of laminar and turbulent regions in Couette flow ${ }^{28}$ represents the coexistence of coherent and incoherent behaviour.

\section{Methods}

Experiments. A population of 40 coupled oscillators is studied in the experiments reported here. The natural period of the uncoupled oscillators is $60.0 \pm 4 \mathrm{~s}$. The oscillations of the particles are accompanied by changes in concentration of the oxidized form of the catalyst $\mathrm{Ru}(\mathrm{bpy})_{3}{ }^{3+}$ and are monitored by the changes in the transmitted light intensity $I$ of each particle. The oscillatory particles are monitored using a CCD (charge-coupled device) camera ${ }^{21}$, with intensity recorded every $3.0 \mathrm{~s}$. With the coupling algorithm switched off, images of the reaction domain are obtained with a background light intensity of $P_{0}$ projected onto each particle. The projected image from a spatial light modulator is reflected with a beam splitter to the reaction domain while permitting intensity measurements with the camera.

An experiment is initiated by applying the intra-group feedback for group A alone $\left(k_{\mathrm{AB}}=0, k_{\mathrm{B}}=0\right)$, allowing this group to fully synchronize. The group $\mathrm{B}$ particles are not coupled and oscillate with approximately random phases. After an initial period, the intra-group feedback for group $\mathrm{B}$, with $k_{\mathrm{B}}=k_{\mathrm{A}}$, and the inter-group feedback, with $k_{\mathrm{AB}}=k_{\mathrm{BA}}$, are simultaneously switched on, and the system is monitored for a further $50 \mathrm{~min}$.

Simulations. A simulation is initiated by synchronizing the oscillators in group A by applying intra-group feedback to that group alone. Intra-group feedback is then applied to the oscillators in group $\mathrm{B}$ at $t=200$, with $k_{\mathrm{B}}=k_{\mathrm{A}}$, where the phase of each oscillator is selected from a random distribution. Inter-group feedback is also applied at this time, with $k_{\mathrm{AB}}=k_{\mathrm{BA}}$. Simulations are typically carried out until $t=100,000$, and the state of the system is then recorded. The model presented here is non-dimensional and we report non-dimensional values; however, dimensional quantities can easily be derived.

The mean amplitude of the chimera is a noisy, flat signal, allowing an empirical threshold in the mean signal amplitude to be established and computationally employed to test for the existence of chimera states and to distinguish the chimera from other states such as semi-synchronized states. A mean signal threshold of 0.35 was found to be satisfactory for detecting the collapse of a chimera state and distinguishing it from other states. The time series in Fig. 3 illustrate the utility of the mean signal threshold for distinguishing the chimera state from other states in the large number of simulations carried out to obtain lifetimes and occurrence probabilities.

Received 19 March 2012; accepted 14 June 2012; published online 15 July 2012

\section{References}

1. Kuramoto, Y. \& Battogtokh, D. Coexistence of coherence and incoherence in nonlocally coupled phase oscillators. Nonlinear Phenom. Complex Syst. 5, 380-385 (2002).

2. Abrams, D. M. \& Strogatz, S. H. Chimera states for coupled oscillators. Phys. Rev. Lett. 93, 174102 (2004). 
3. Shima, S. \& Kuramoto, Y. Rotating spiral waves with phase-randomized core in nonlocally coupled oscillators. Phys. Rev. E 69, 036213 (2004).

4. Abrams, D. M. \& Strogatz, S. H. Chimera states in a ring of nonlocally coupled oscillators. Int. J. Bifurc. Chaos 16, 21-37 (2006).

5. Abrams, D. M., Mirollo, R., Strogatz, S. H. \& Wiley, D. A. Solvable model for chimera states of coupled oscillators. Phys. Rev. Lett. 101, 084103 (2008).

6. Sethia, G. C., Sen, A. \& Atay, F. M. Clustered chimera states in delay-coupled oscillator systems. Phys. Rev. Lett. 100, 144102 (2008).

7. Omel'chenko, O. E., Maistrenko, Y. L. \& Tass, P. A. Chimera states: The natural link between coherence and incoherence. Phys. Rev. Lett. 100, 044105 (2008).

8. Laing, C. R. Chimera states in heterogeneous networks. Chaos 19, 013113 (2009).

9. Bordyugov, G., Pikovsky, A. \& Rosenblum, M. Self-emerging and turbulent chimeras in oscillator chains. Phys. Rev. E 82, 035205 (2010)

10. Martens, E. A., Laing, C. R. \& Strogatz, S. H. Solvable model of spiral wave chimeras. Phys. Rev. Lett. 104, 044101 (2010).

11. Shanahan, M. Bistable chimera attractors on a triangular network of oscillator populations. Chaos 20, 013108 (2010).

12. Martens, E. A. Metastable chimera states in community-structured oscillator networks. Phys. Rev. E 82, 016216 (2010).

13. Laing, C. R. Chimeras in networks of planar oscillators. Phys. Rev. E 81, 066221 (2010).

14. Martens, E. A. Chimeras in a network of three oscillator populations with varying network topology. Chaos 20, 3499502 (2010).

15. Motter, A. E. Spontaneous synchrony breaking. Nature Phys. 6, 164-165 (2010).

16. Omelchenko, I., Maistrenko, Y., Hovel, P. \& Schöll, E. Loss of coherence in dynamical networks: spatial chaos and chimera states. Phys. Rev. Lett. 106, $234102(2011)$.

17. Wolfrum, M. \& Omel'chenko, O. Chimera states are chaotic transients. Phys. Rev. E 84, 015201 (2011).

18. Lee, W. S., Restrepo, J. G., Ott, E. \& Antonsen, T. M. Dynamics and pattern formation in large systems of spatially-coupled oscillators with finite response times. Chaos 21, 023122 (2011)

19. Laing, C. R., Rajendran, K. \& Kevrekidis, I. G. Chimeras in random noncomplete networks of phase oscillators. Chaos 22, 013132 (2012).

20. Zaikin, A. N. \& Zhabotinsky, A. M. Concentration wave propagation in twodimensional liquid-phase self-oscillating system. Nature 225, 535-537 (1970).
21. Taylor, A. F. et al. Clusters and switchers in globally coupled photochemical oscillators. Phys. Rev. Lett. 100, 214101 (2008).

22. Kuhnert, L. A new optical photochemical memory device in a light-sensitive chemical active medium. Nature 319, 393-394 (1986).

23. Pikovsky, A., Rosenblum, M. \& Kurths, J. Synchronization: A Universal Concept in Nonlinear Sciences (Cambridge Univ. Press, 2003).

24. Golomb, D., Hansel, D., Shraiman, B. \& Sompolinsky, H. Clustering in globally coupled phase oscillators. Phys. Rev. A 45, 3516-3530 (1992).

25. Zhabotinsky, A. M., Bucholtz, F., Kiyatkin, A. B. \& Epstein, I. R. Oscillations and waves in metal-ion-catalyzed bromate oscillating reactions in highly oxidized states. J. Phys. Chem. 97, 7578-7584 (1993).

26. Taylor, A. F., Tinsley, M. R., Wang, F. \& Showalter, K. Phase clusters in large populations of chemical oscillators. Angew. Chem. Int. Edn. 50, 10161-10164 (2011).

27. Rattenborg, N. C., Amlaner, C. J. \& Lima, S. L. Behavioral, neurophysiological and evolutionary perspectives on unihemispheric sleep. Neurosci. Biobehav. Rev. 24, 817-842 (2000).

28. Barkley, D. \& Tuckerman, L. S. Computational study of turbulent laminar patterns in Couette flow. Phys. Rev. Lett. 94, 014502 (2005).

\section{Acknowledgements}

We thank the National Science Foundation (No. CHE-0809058) for supporting this research.

\section{Author contributions}

M.R.T. and S.N. performed the experiments and simulations of this study. M.R.T., S.N and K.S. designed the study, and M.R.T. and K.S. wrote the paper. All authors discussed the results and commented on the manuscript.

\section{Additional information}

Supplementary information is available in the online version of the paper. Reprints and permissions information is available online at www.nature.com/reprints. Correspondence and requests for materials should be addressed to K.S.

\section{Competing financial interests}

The authors declare no competing financial interests. 